\title{
The Efficacy of Coaching Approach Behavior and Leading by Modeling on Separation Anxiety Disorder among Preschool Children
}

\author{
Mohammad Mohammadpour*, Khalil Esmailpour \\ Department of Psychology, Faculty of Education and Psychology, Tabriz University, Tabriz, Iran
}

\section{ABSTRACT}

Introduction: Separation anxiety is the most common anxiety disorder in children. The neglect of treatment of this disorder at early ages could be a risk factor for other childhood and adult psychological disorders. The purpose of this study was to examine the efficacy of Coaching Approach Behavior and Leading by Modeling (CALM Program) on reduction of the symptoms of separation anxiety disorder among preschool children. Materials and Methods: The research plan was designed as pretest-posttest with control group. The sample included 30 children with separation anxiety disorder. Children were selected by multi-stage cluster sampling method and assigned randomly in the experimental and control groups (each group 15 individuals). CALM-based treatment program for each participant of experimental group was conducted across 12 sessions, in the form of mother-child interaction. Spence Children's Anxiety Scale (parent form) was used to measure the severity of separation anxiety disorder. Results: CALM-based treatment interventions created a significant reduction in the symptoms of separation anxiety in the experimental group compared to the control group on the post-test and follow-up stages. Conclusion: CALM-based treatment interventions can be used as applicable and effective treatment for reduction of the symptoms of separation anxiety in preschooler children.

\section{Key words:}

1. Anxiety, Separation

2. Mother-Child Relations

3. Psychotherapy

*Corresponding Author: Mohammad Mohammadpour

E-mail: m.mohammadpour1989@gmail.com

doi: 10.18869/acadpub.shefa.5.1.29 


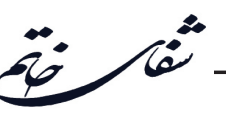

\section{اثربخشى برنامهٔ مربيگرى رفتار و هدايت از طريق مدلسازى بر اختلال اضطراب جدايى كودكان ييش دبستانى}

محمد محمديور"، خليل اسماعيل يور

كروه روانشناسى، دانشكده روانشناسى و علوم تربيتى، دانشًاه تبريز، تبريز، ايران

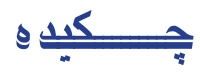

مقدمه: اضطراب جدايى شايعترين اختلال اضطرابى در كودكان است. عدم توجه به درمان بران اين اختلال

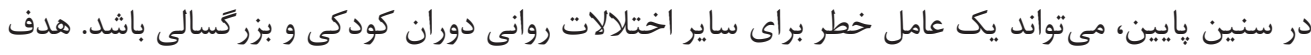

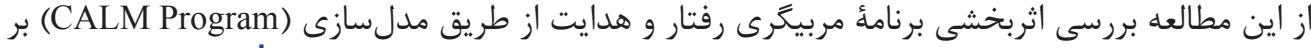

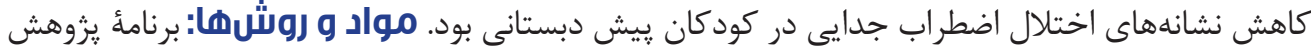

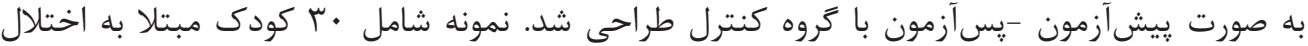

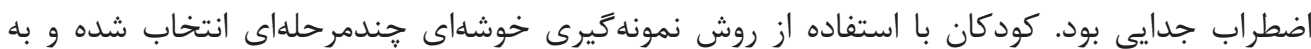

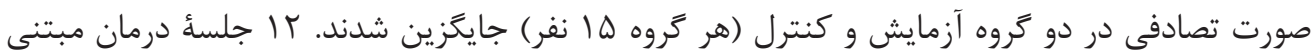

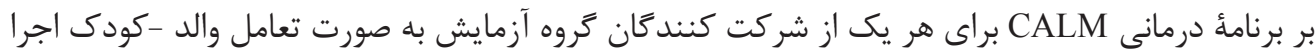

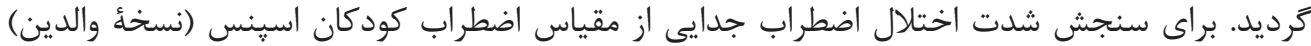

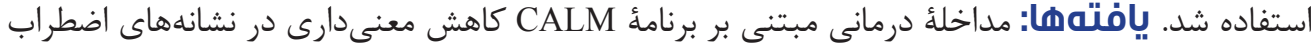

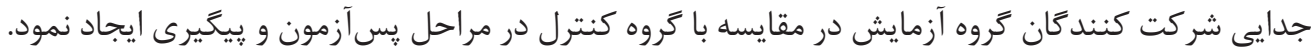

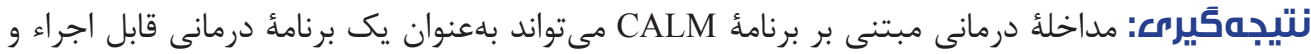

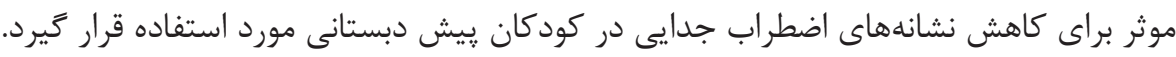

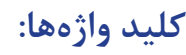

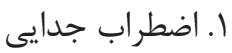

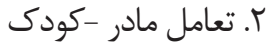

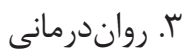

" نويسنده مسئول: محمد محمديور آدرس الكترونيكى: m.mohammadpour1989@gmail.com 


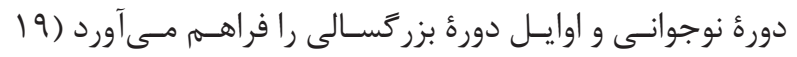

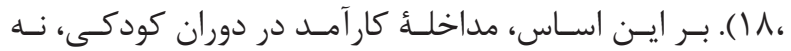

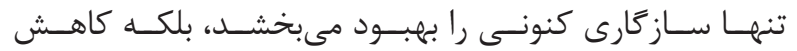

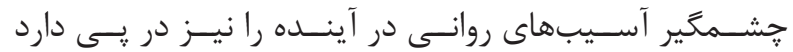

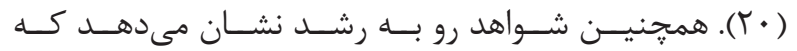

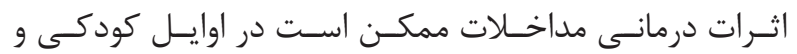

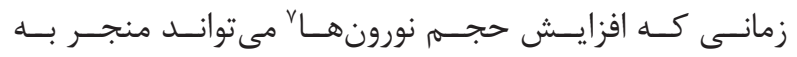

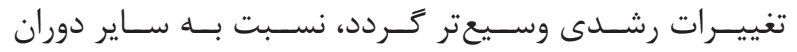

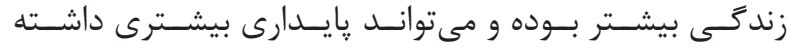

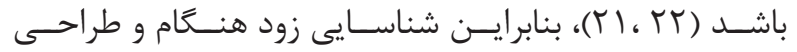

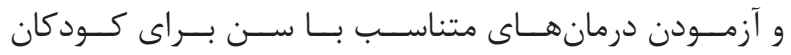

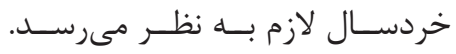

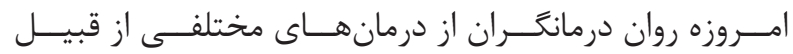

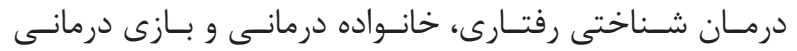

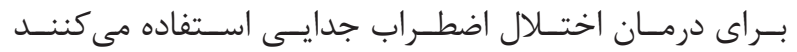

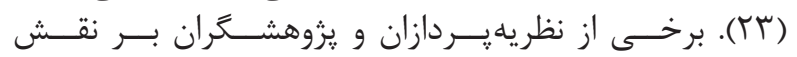

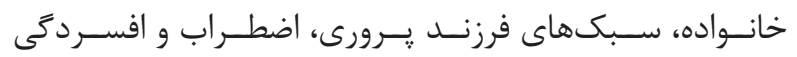

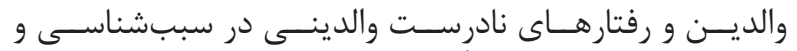

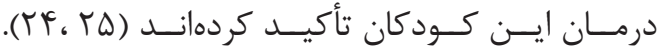

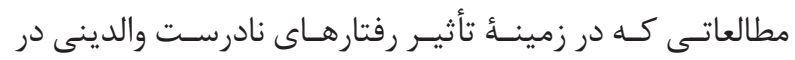

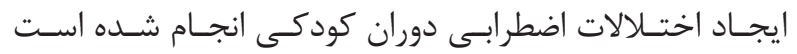

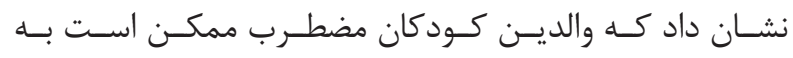

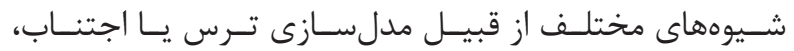

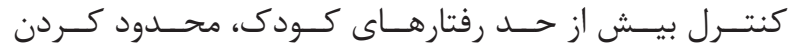

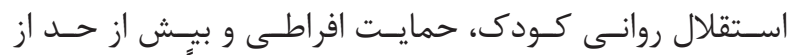

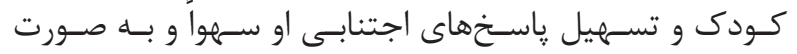

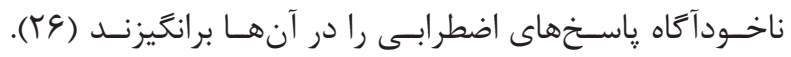

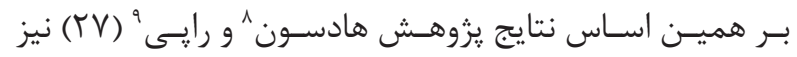

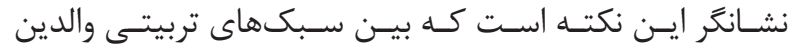

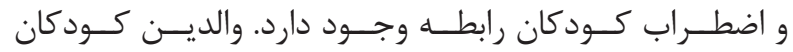

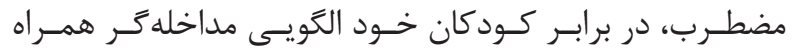

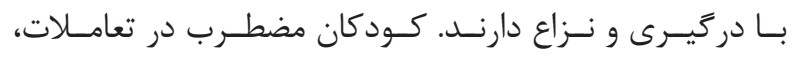

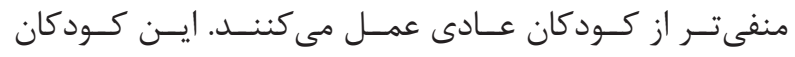

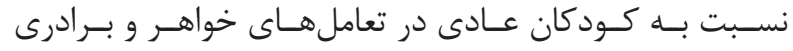

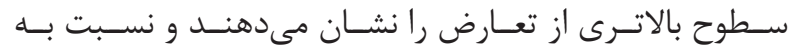

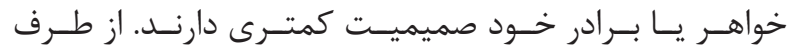

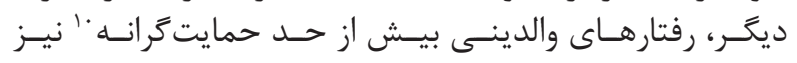

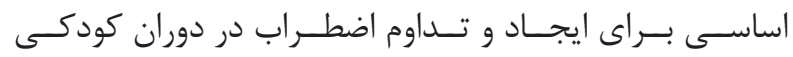

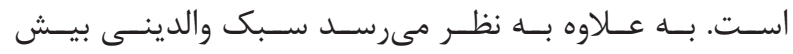

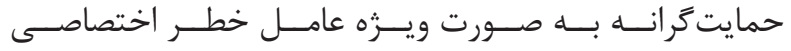

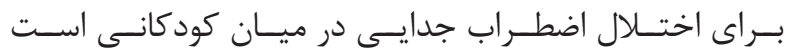

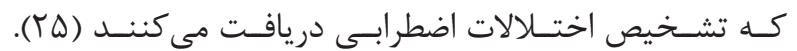

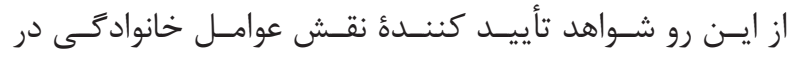

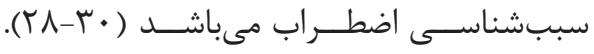

${ }^{1}$ Separation anxiety disorder

${ }^{2}$ Eating disorders

${ }^{3}$ Depression

${ }^{4}$ Somatic disorders

${ }^{5}$ Alcohol abuse

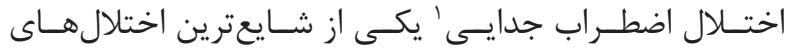

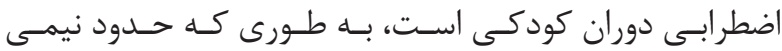

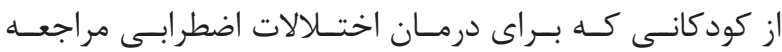

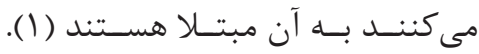

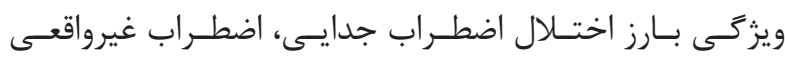

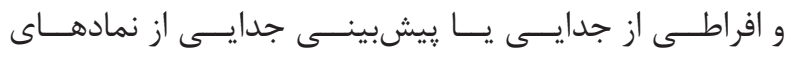

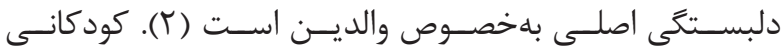

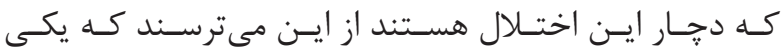

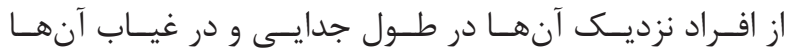

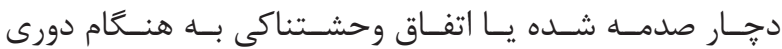

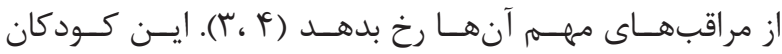

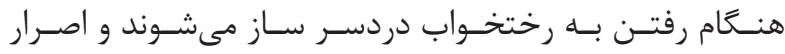

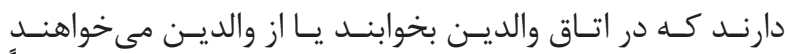

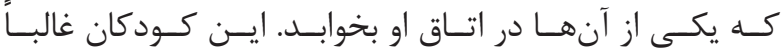

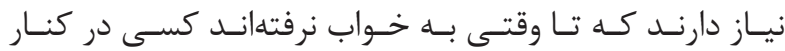

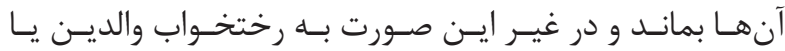

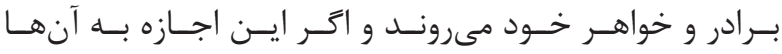

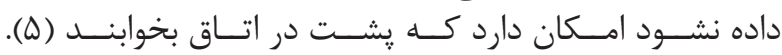

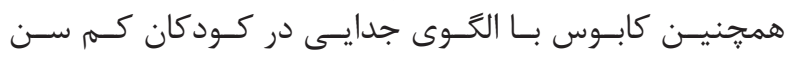

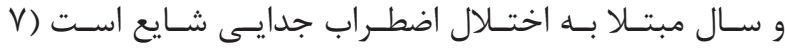

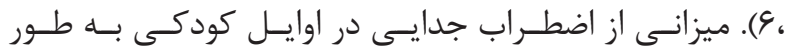

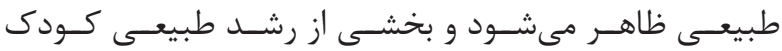

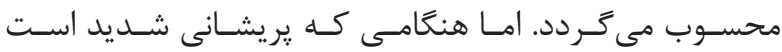

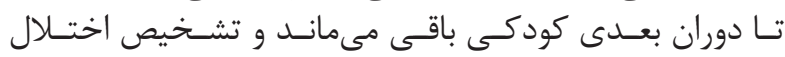

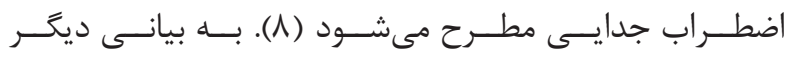

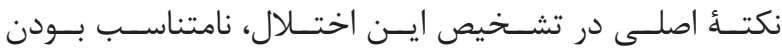

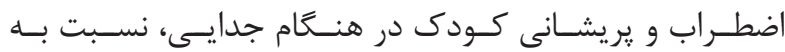

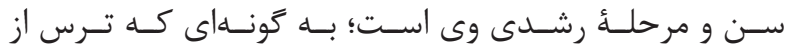

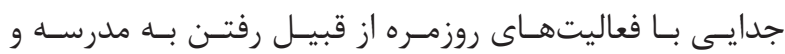

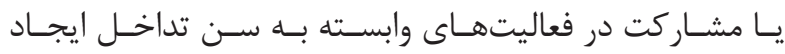

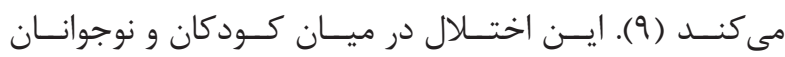

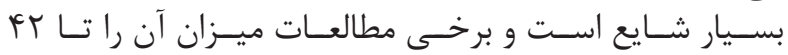

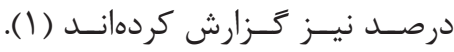

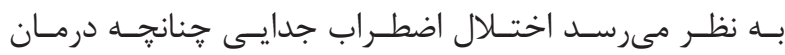

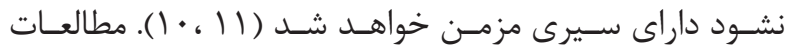

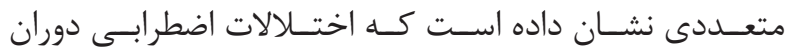

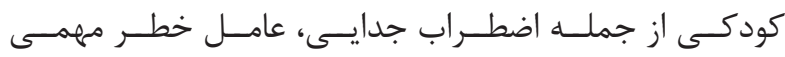

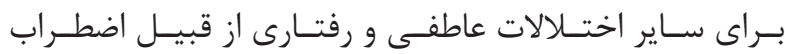

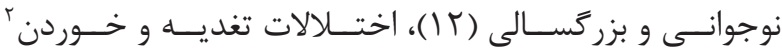

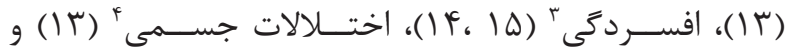

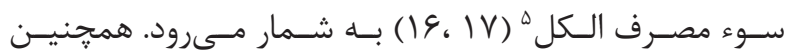

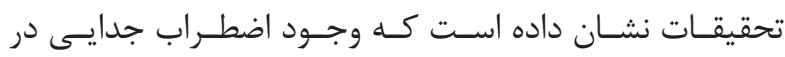

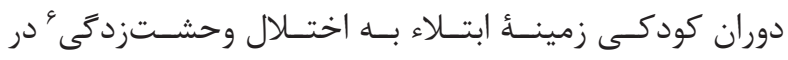

\footnotetext{
${ }^{6}$ Panic disorders

${ }^{7}$ Neuroplasticity

${ }^{8}$ Hudson

${ }^{9}$ Rapee

${ }^{10}$ Overprotective
} 


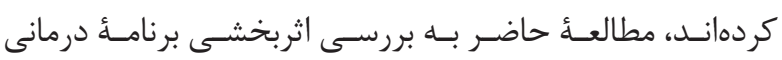

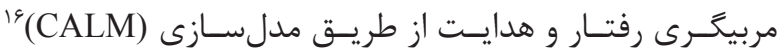

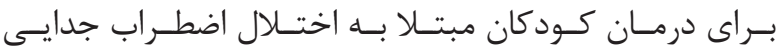

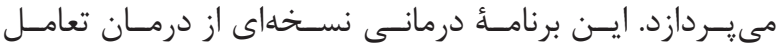

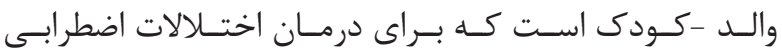

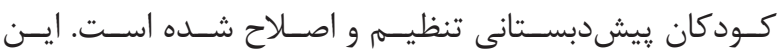

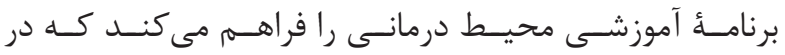

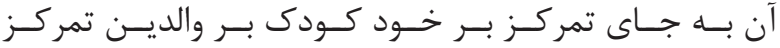

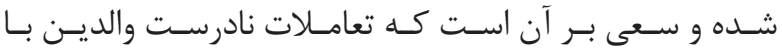

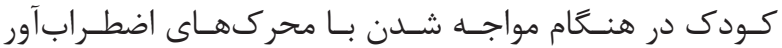
شناسـايى و اصـلاح كـردد (T) (F).

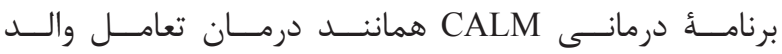

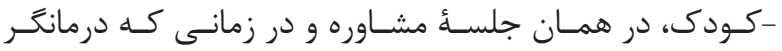

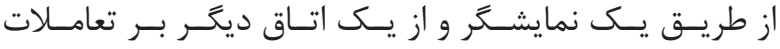

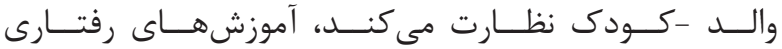

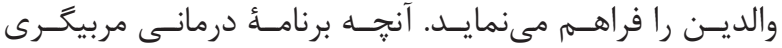

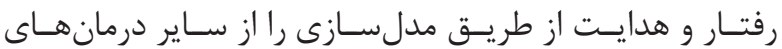

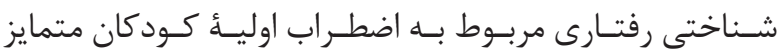

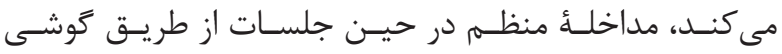

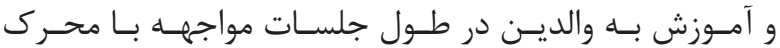

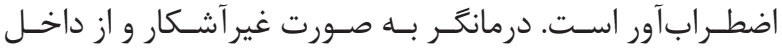

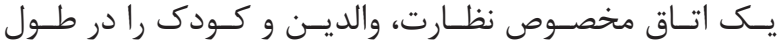

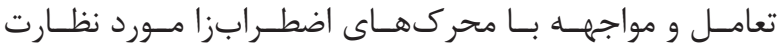

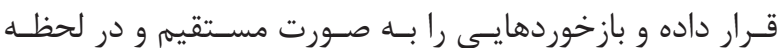

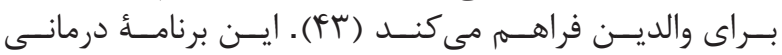

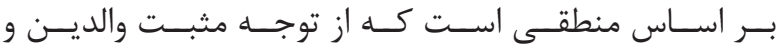

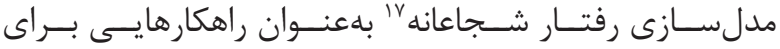

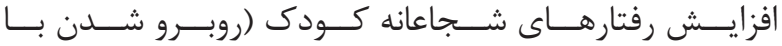

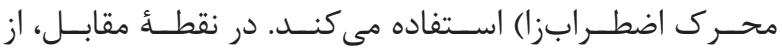

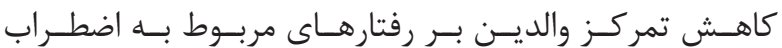

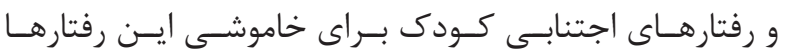

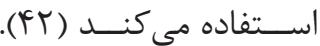

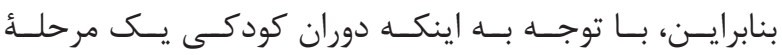

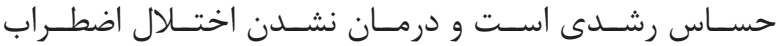

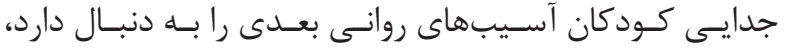

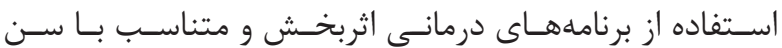

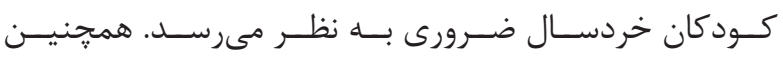

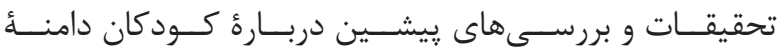

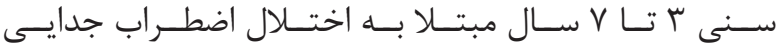

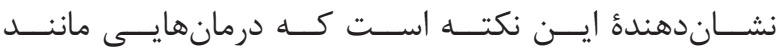

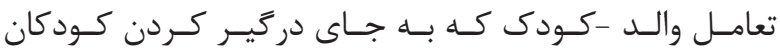

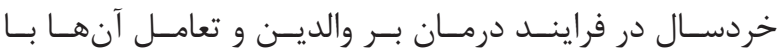

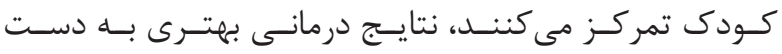

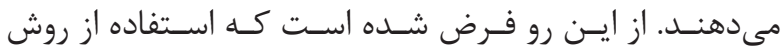

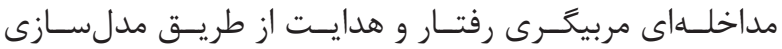

${ }^{11}$ Parent child interaction therapy

${ }^{12}$ Eyberg

${ }^{13}$ Choate

${ }^{14}$ Pincus

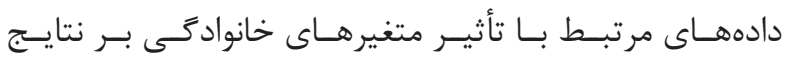

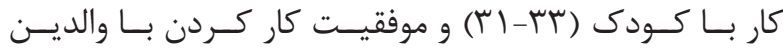

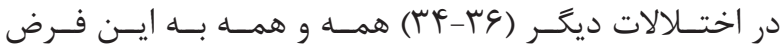

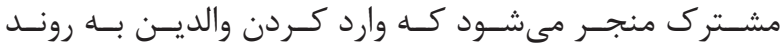

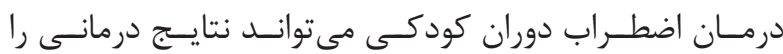

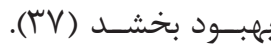

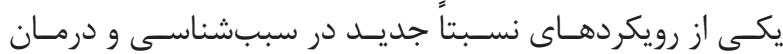

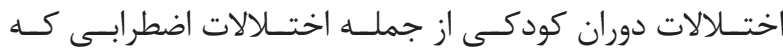

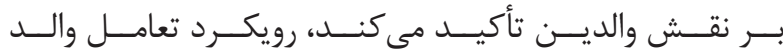

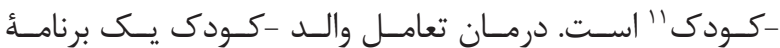

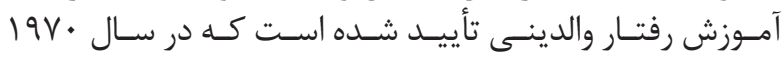

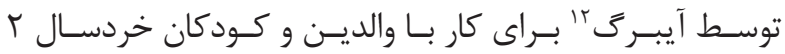

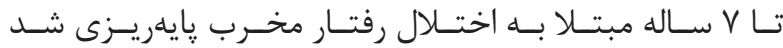

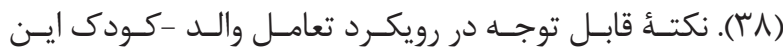

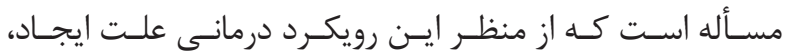

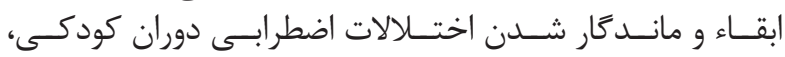

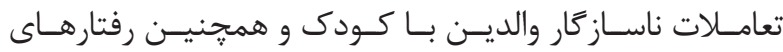

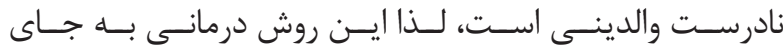

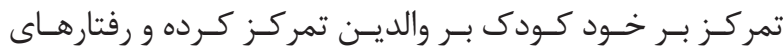

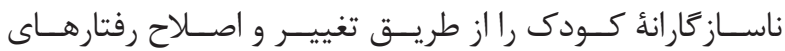

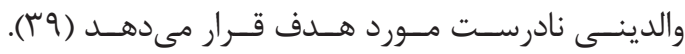

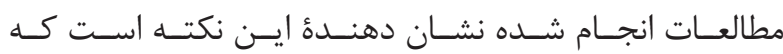

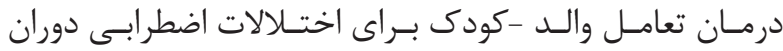

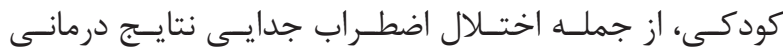

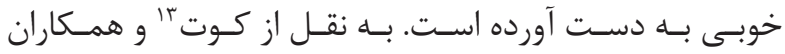

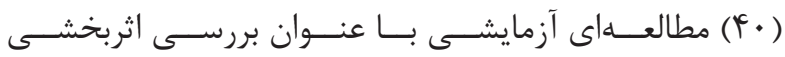

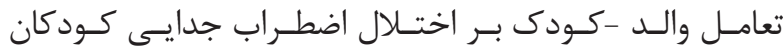

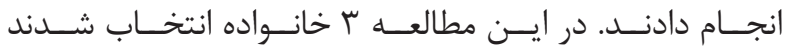

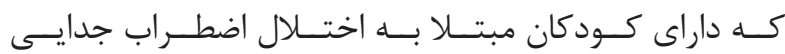

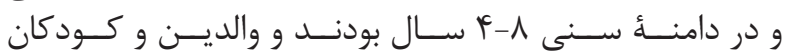

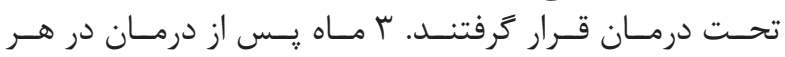

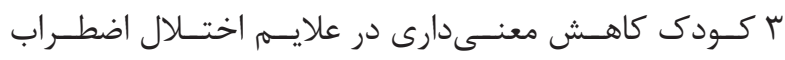

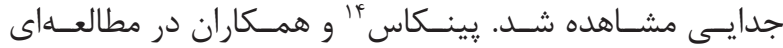

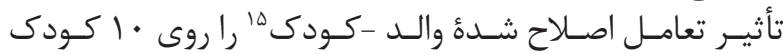

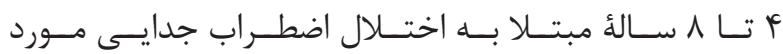

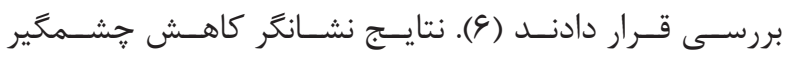

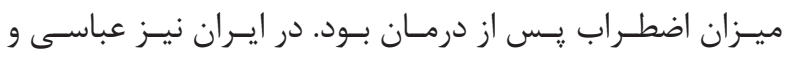

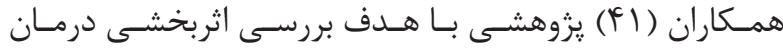

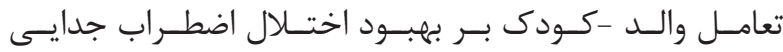

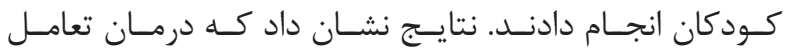

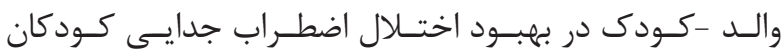
اثربخــش اسـت

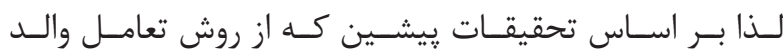

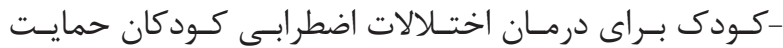

${ }^{15}$ Modified parent-child interaction therapy

${ }^{16}$ Coaching approach behavior and leading by modeling

${ }^{17}$ Brave 


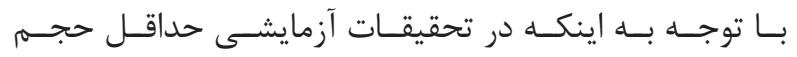

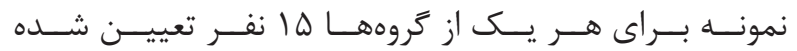

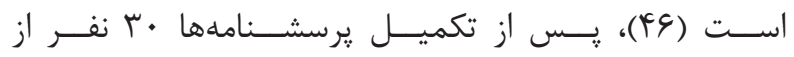

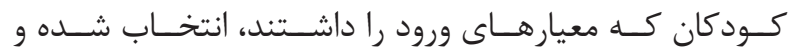

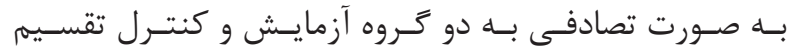

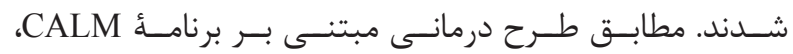

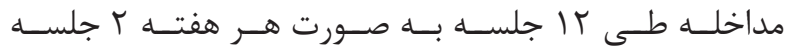

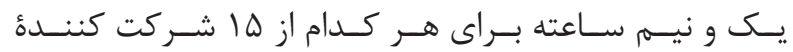

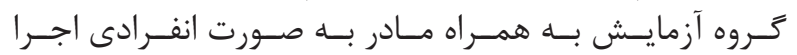

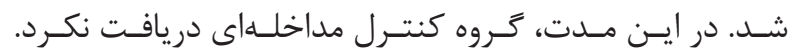

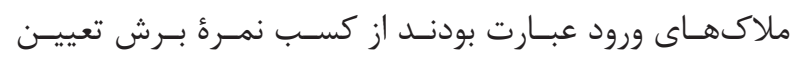

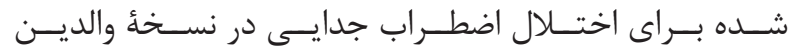

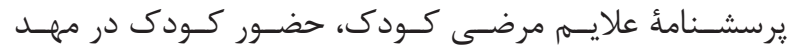

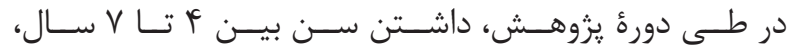

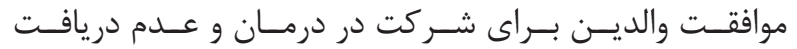

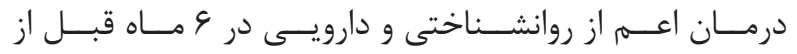

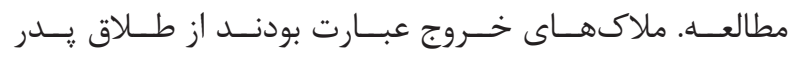

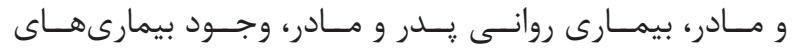

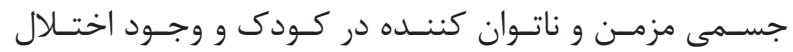

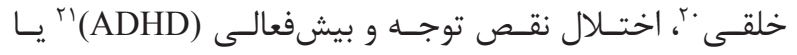

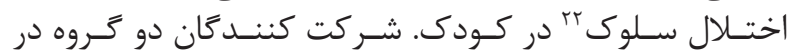

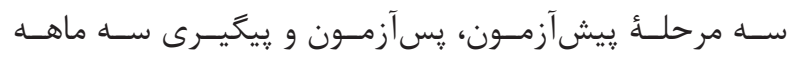

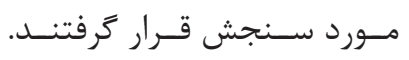

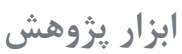

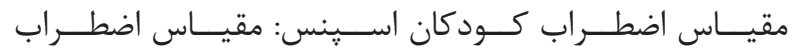

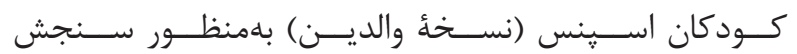

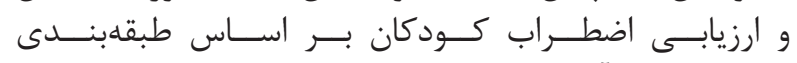

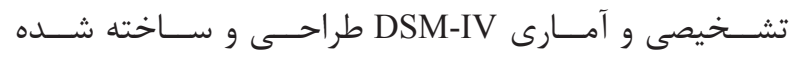

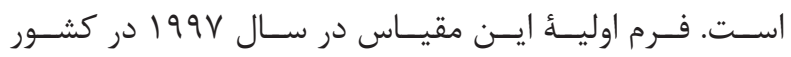

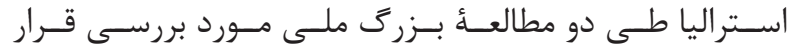

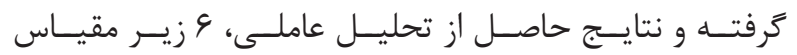

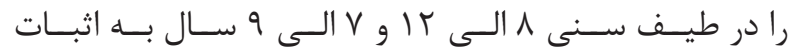

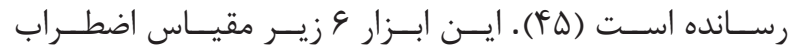

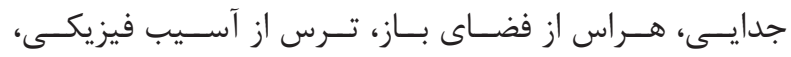

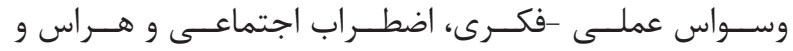

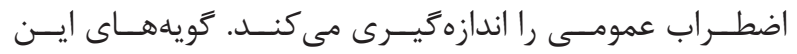

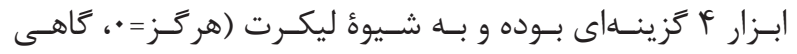

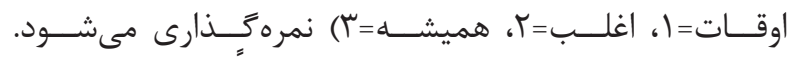

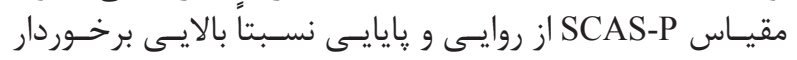

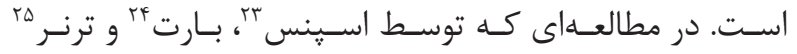

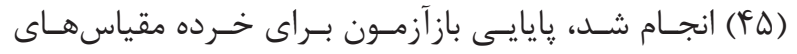

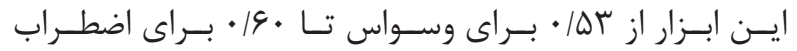

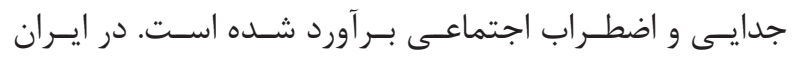

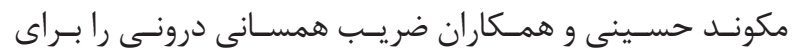

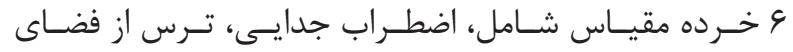

${ }^{18}$ Children symptoms inventory- 4

${ }^{19}$ Spence children anxiety scale- parent form

${ }^{20}$ Mood disorder

${ }^{21}$ Attention deficit hyperactivity disorder

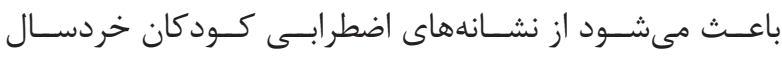

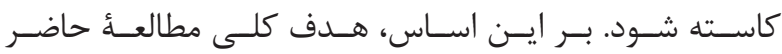

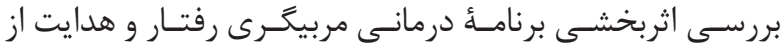

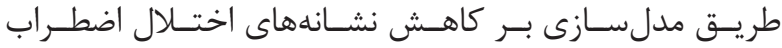

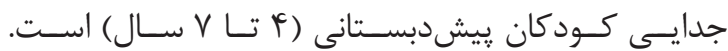

مواد و روشها

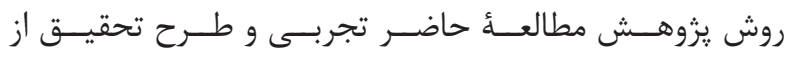

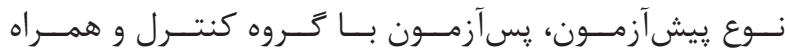

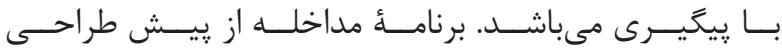

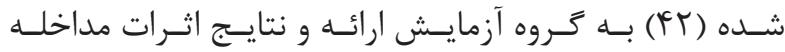

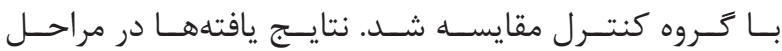

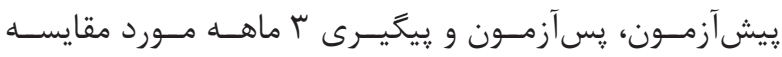

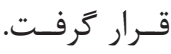

شر كت كنند

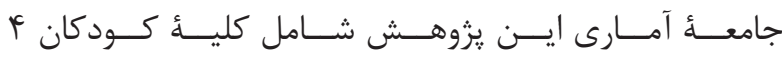

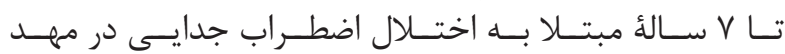

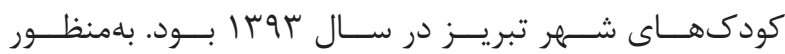

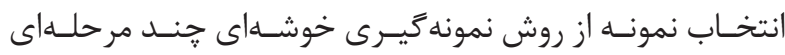

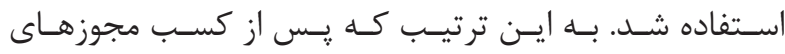

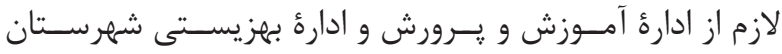

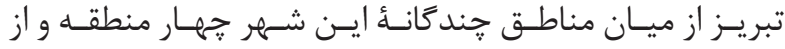

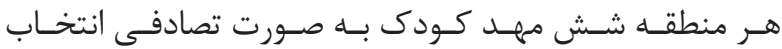

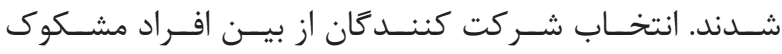

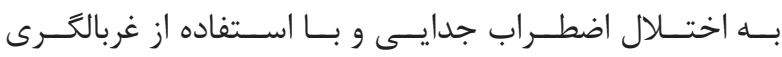

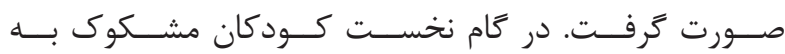

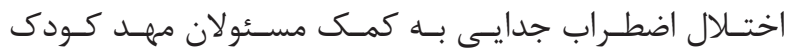

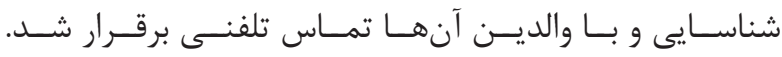

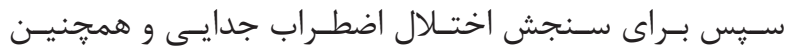

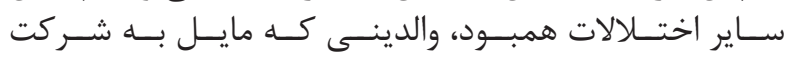

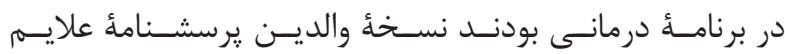

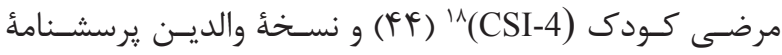

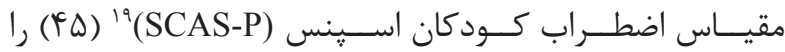

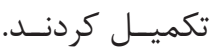

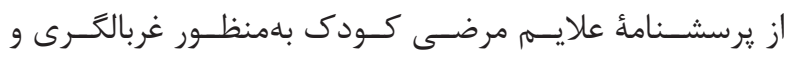

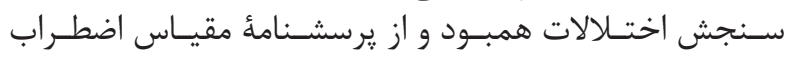

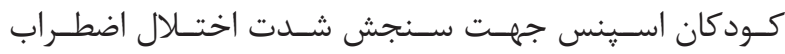

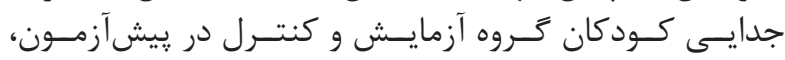

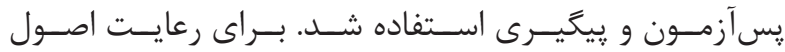

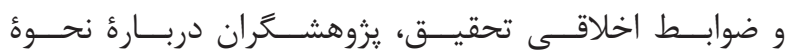

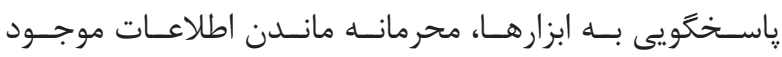

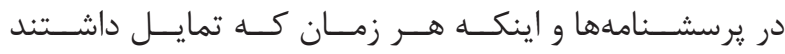

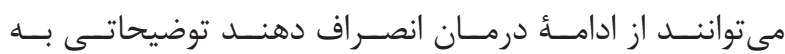

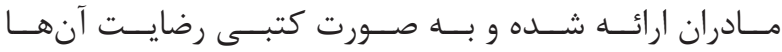

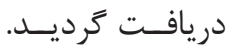

${ }^{22}$ Conduct disorder

${ }^{23}$ Spence

${ }^{24}$ Barrett

${ }^{25}$ Turner 


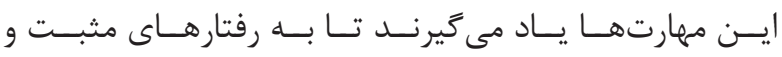

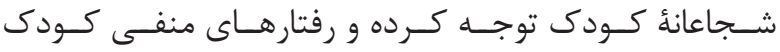

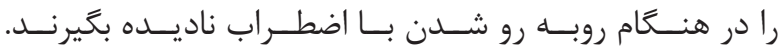

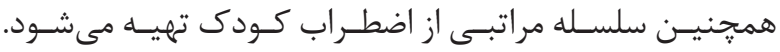

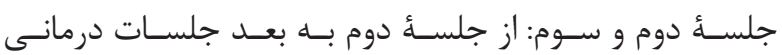

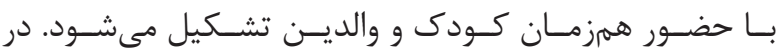

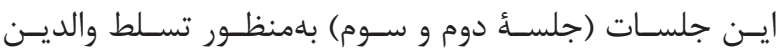

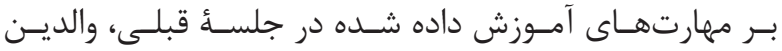

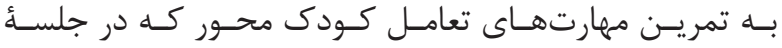

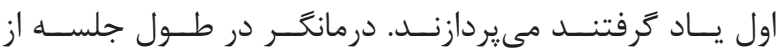

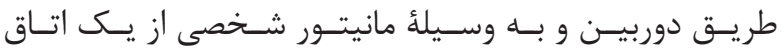

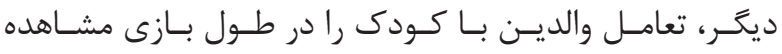

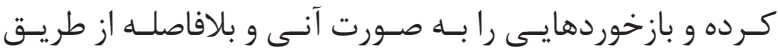

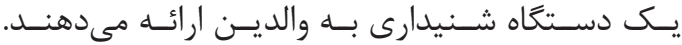

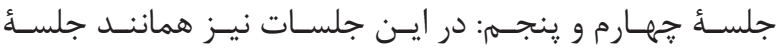

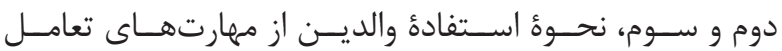

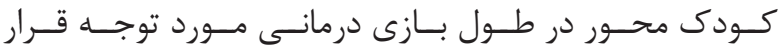

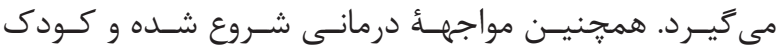

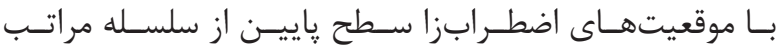

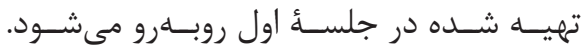

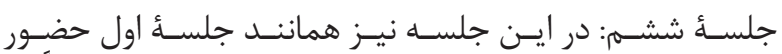

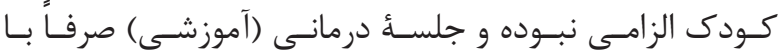

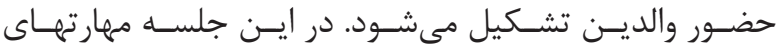

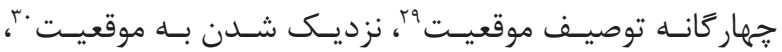

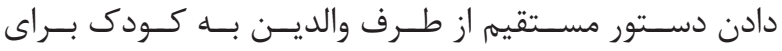

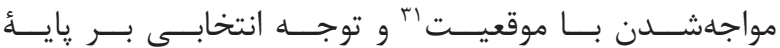

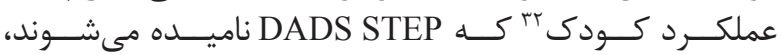

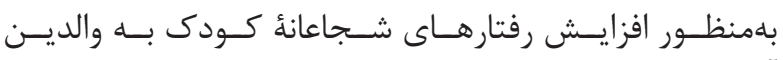

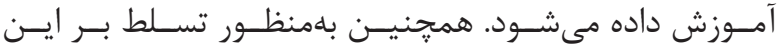

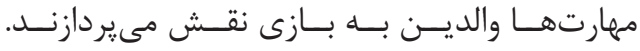

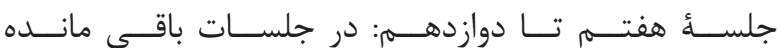

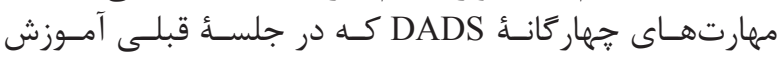

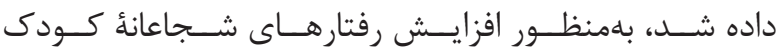

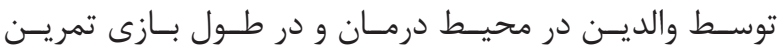

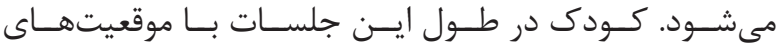

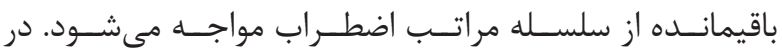

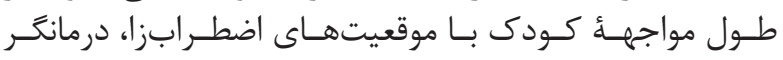

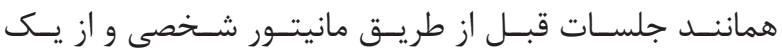

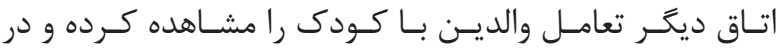

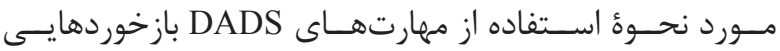

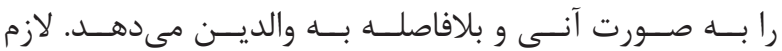

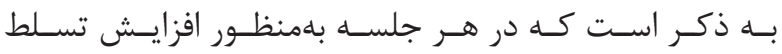

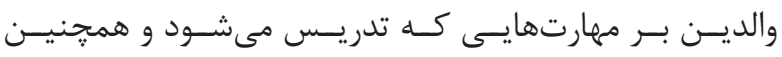

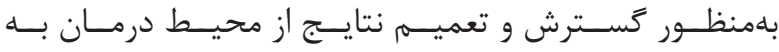

\footnotetext{
${ }^{26}$ Sprafkin, Loney Unita \& Gadow

${ }^{27}$ Puliafico, Comer \& Albano

${ }^{28}$ Child directed interaction
}

${ }^{29}$ Describe situation

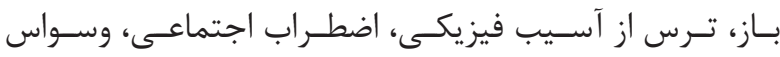

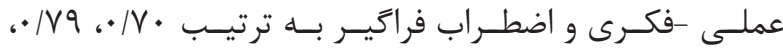

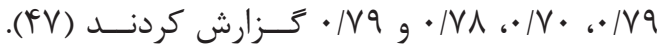

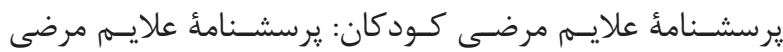

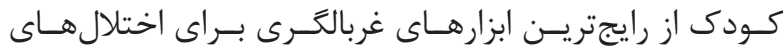

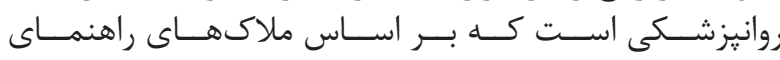

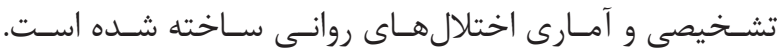

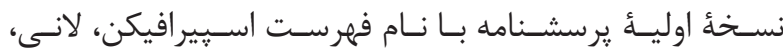

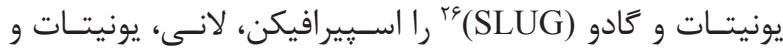

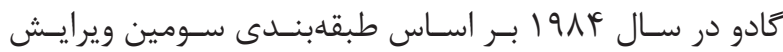

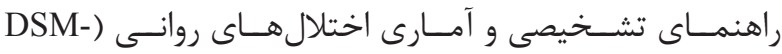
(III

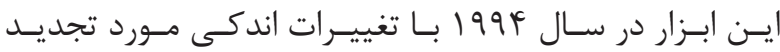

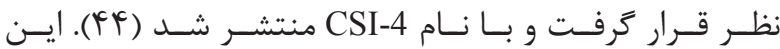

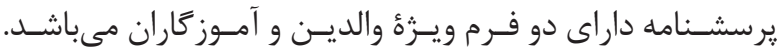

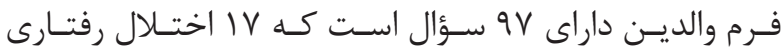

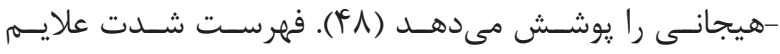

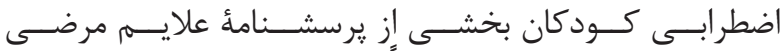

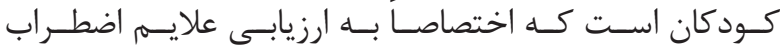

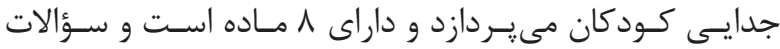

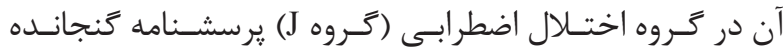

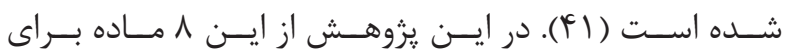

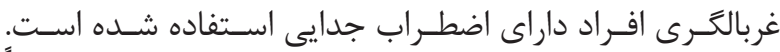

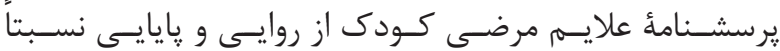

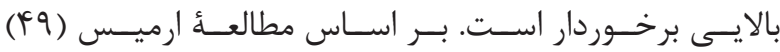

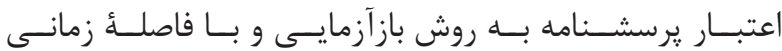

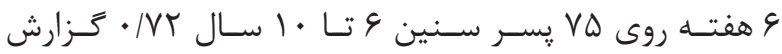

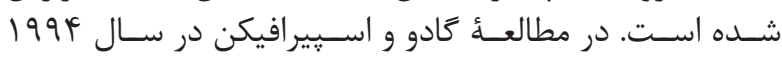

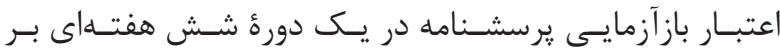

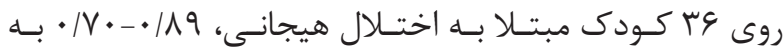

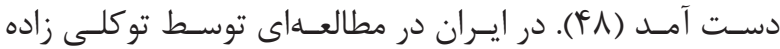

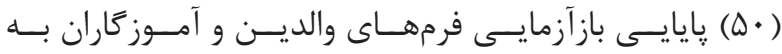

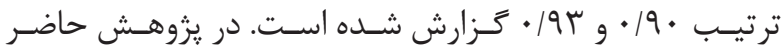

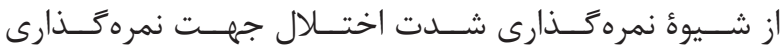

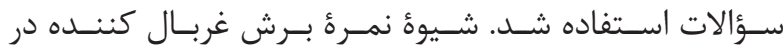

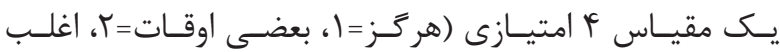

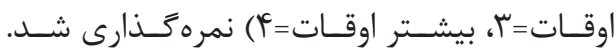

برنامةٔ مداخله درمانى (طرح درمان)

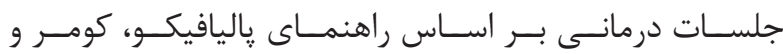

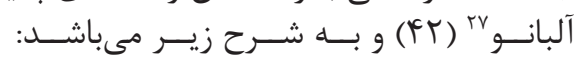

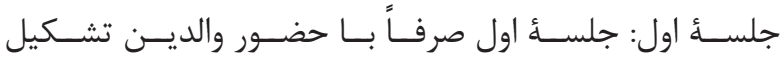

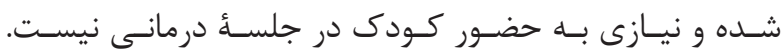

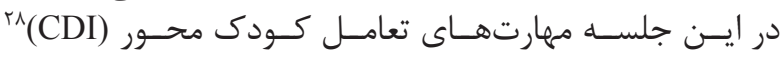

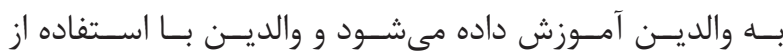

\footnotetext{
${ }^{30}$ Approach situation

${ }^{31}$ Give direct command for child to join situation

${ }^{32}$ Provide selective attention based on child performance
} 


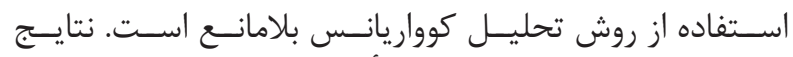

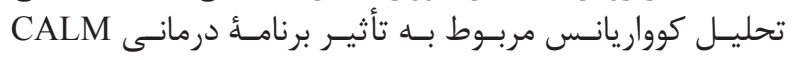

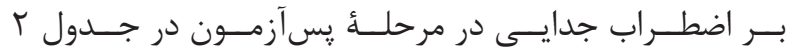

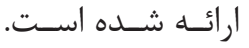

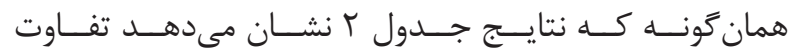

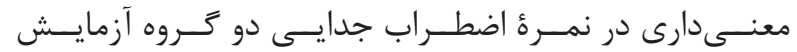

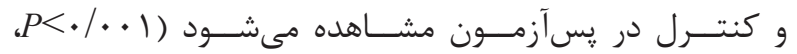

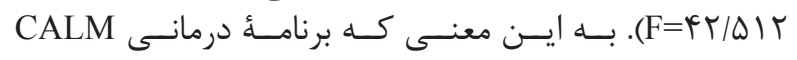

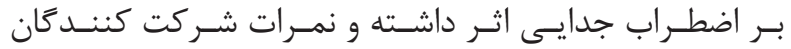

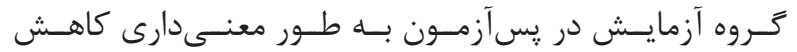

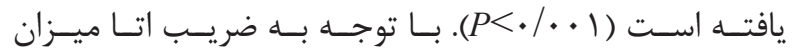

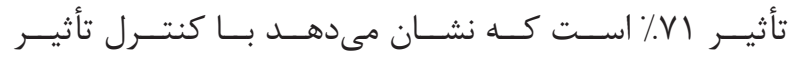

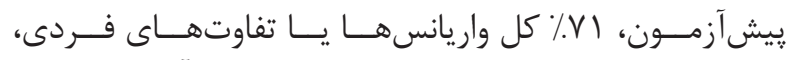

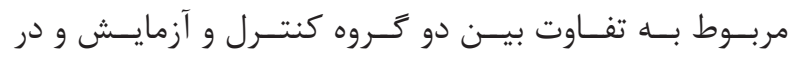

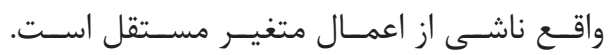

\section{بحث و نتيجه كيرى}

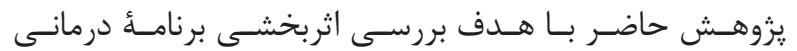

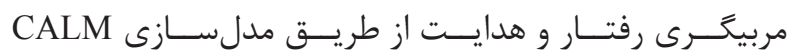

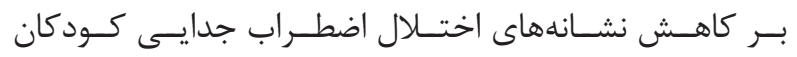

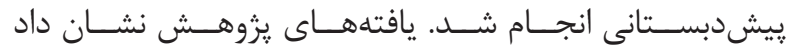

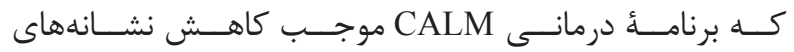

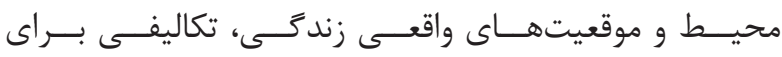

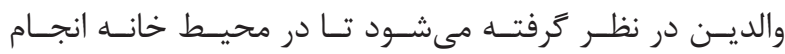

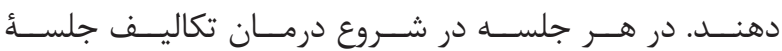

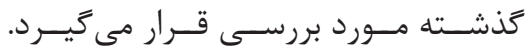

$$
\text { آناليز آمارى }
$$

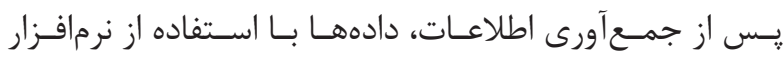

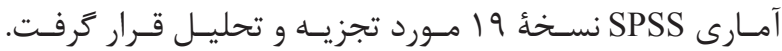

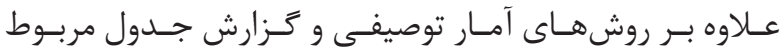

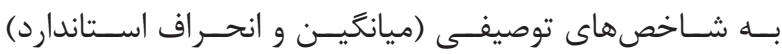

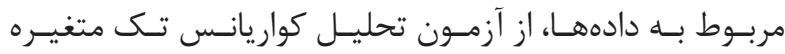

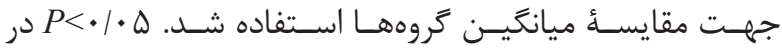

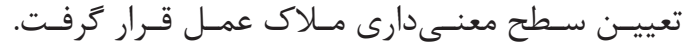

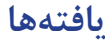

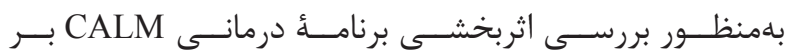

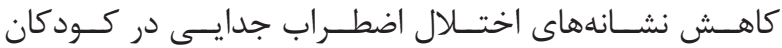

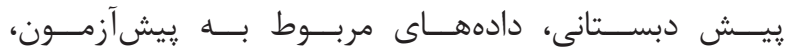

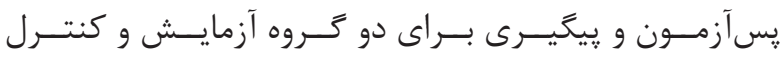

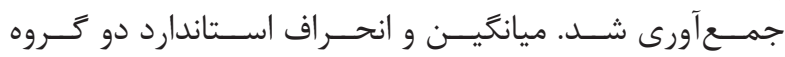

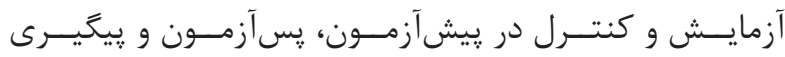

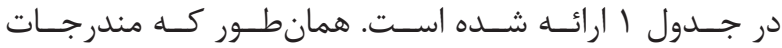

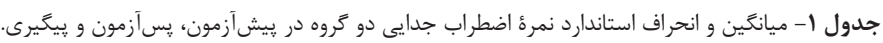

\begin{tabular}{|c|c|c|c|}
\hline ييكيرى & يس آزمون & بيش آزمون & كروه \\
\hline$r / A \pm 1 / T r$ & $\Delta / \Lambda \pm 1 / \Gamma)$ & $1 / / \Delta \pm 1 / r \Delta^{\mathrm{a}}$ & آزمايش \\
\hline$N / \Lambda \pm 1 / \cdot r$ & $9 / 1 \pm 1 / 19$ & $\| / \Lambda \pm V / r$ & كنترل \\
\hline
\end{tabular}

جدول r- نتايج تحليل كوواريانس تأثير برنامٔ درمانى CALM بر كاهش اضطراب جدايى كودكان.

\begin{tabular}{|c|c|c|c|c|c|}
\hline مجذور اتا & معنى & $\mathbf{F}$ & ميانكين مجذورات & درجه آزادى & منابع تغييرات \\
\hline$\cdot / 19$ & $\cdot|1|$ & T/FYA & $4 / 1 \cdot 9$ & 1 & بيش آزمون \\
\hline$\cdot / V I$ & $\ldots$ & ET/DIT & $\Delta Q / T \Delta T$ & 1 & عضويت كروهى \\
\hline
\end{tabular}

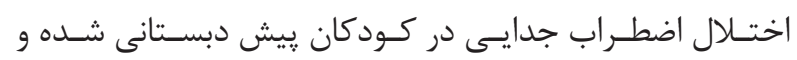

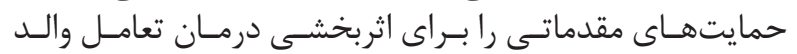

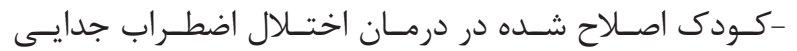

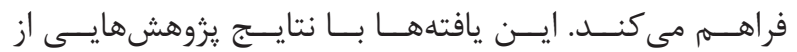

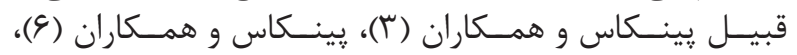

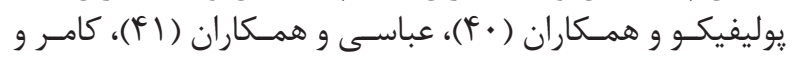

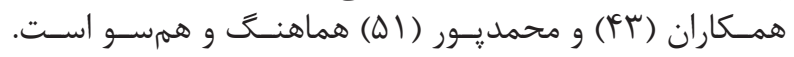

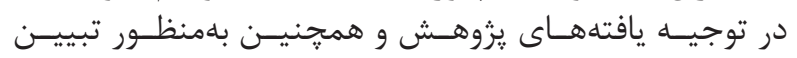

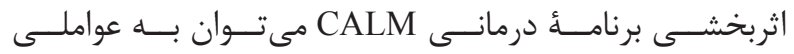

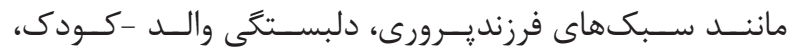

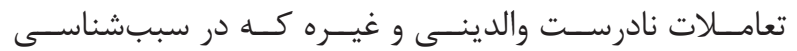

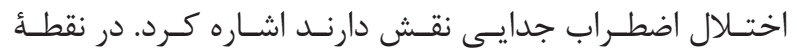

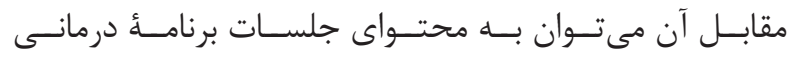

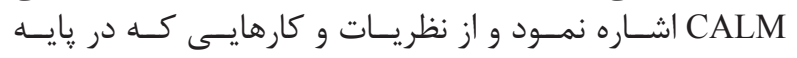

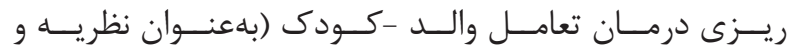

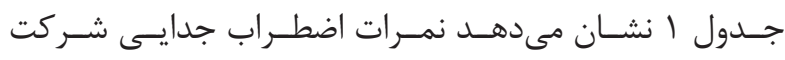

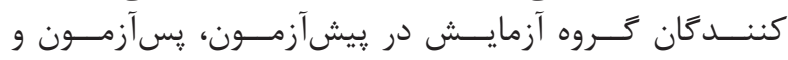

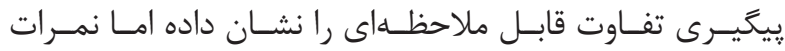

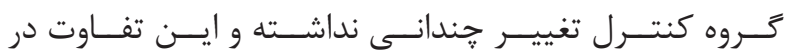

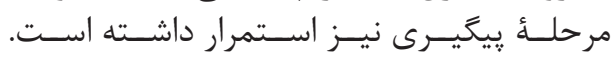

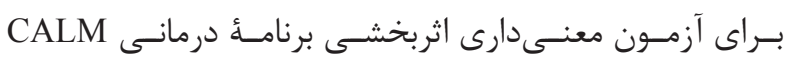

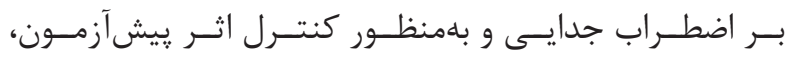

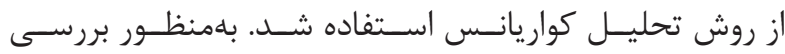

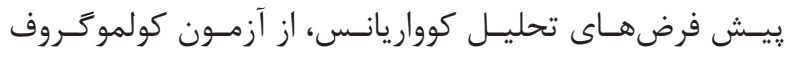

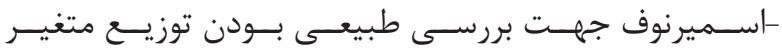

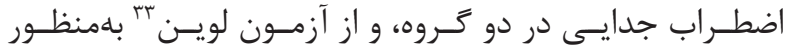

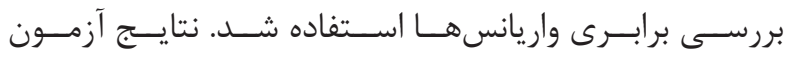

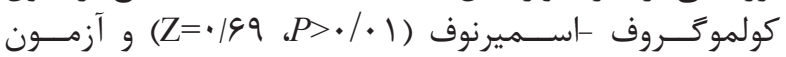

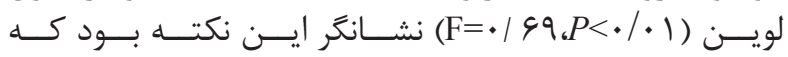

${ }^{33}$ Levene's test 


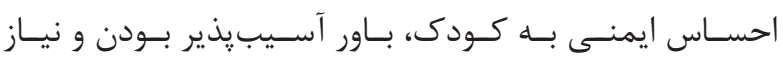

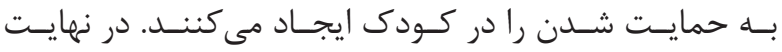

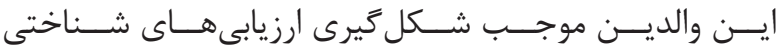

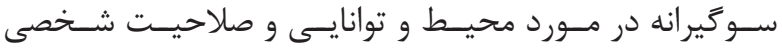

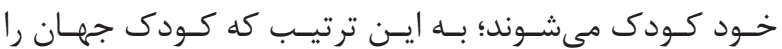

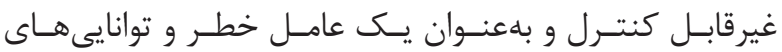

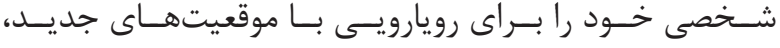

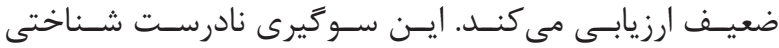

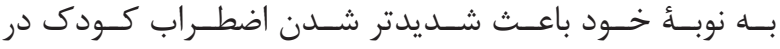

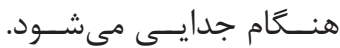

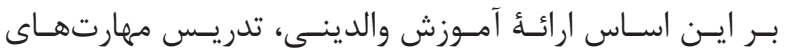

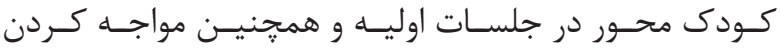

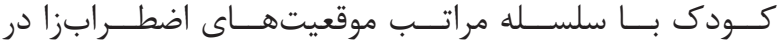

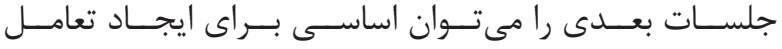

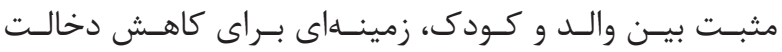

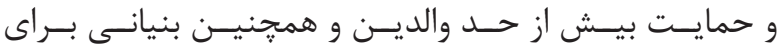

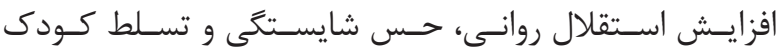

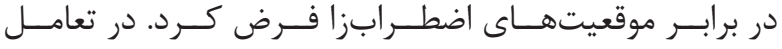

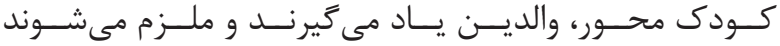

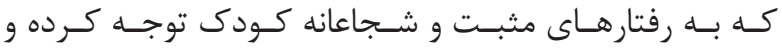

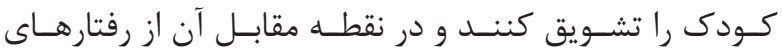

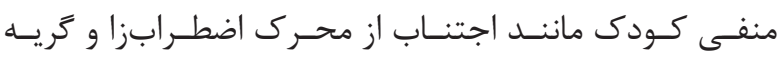

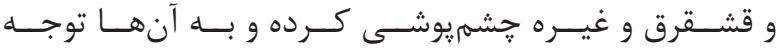

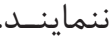

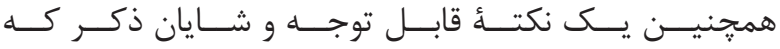

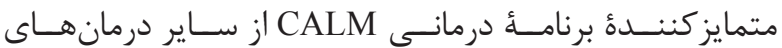

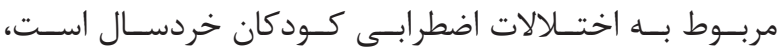

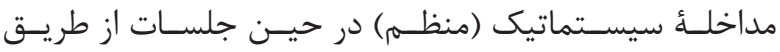

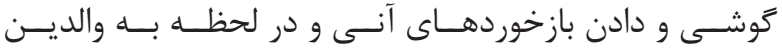

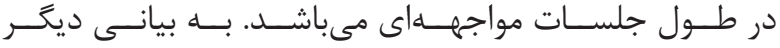

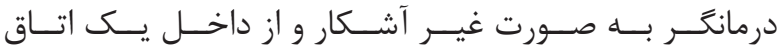

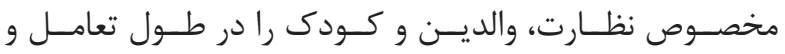

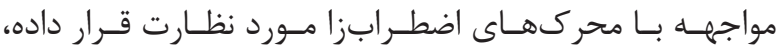

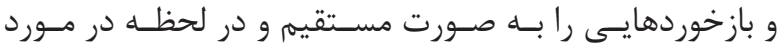

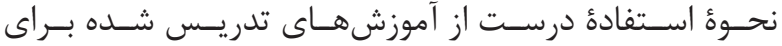

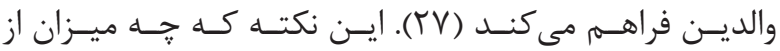

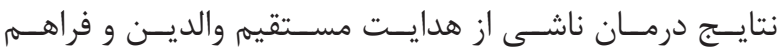

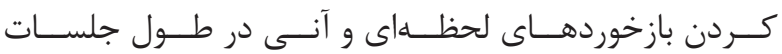

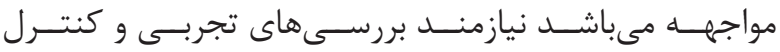

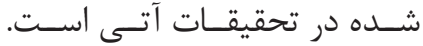

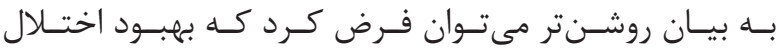

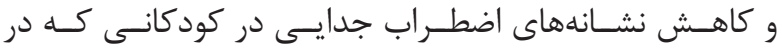

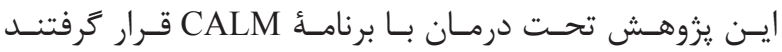

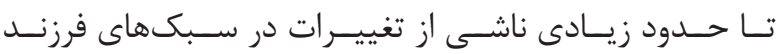

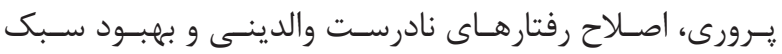

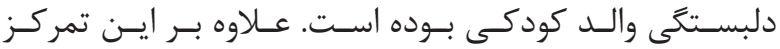

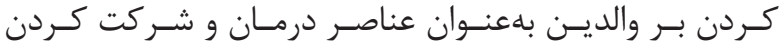

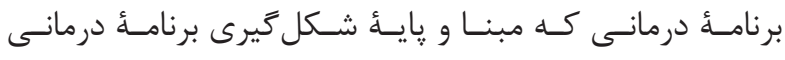

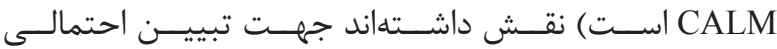

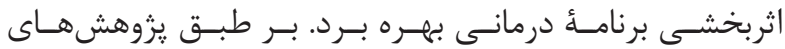

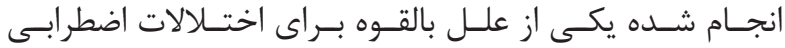

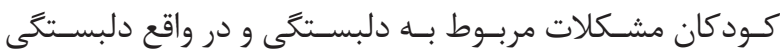

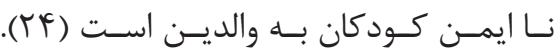

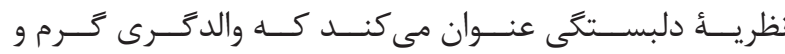

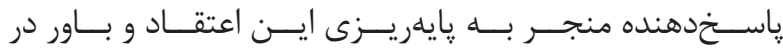

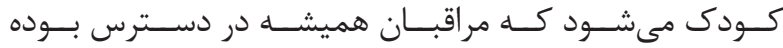

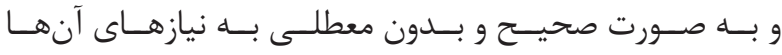

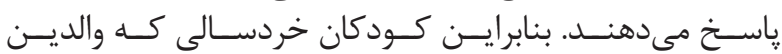

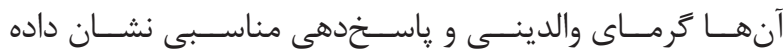

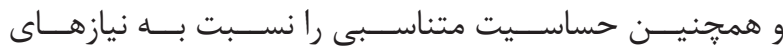

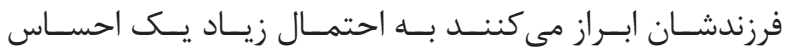

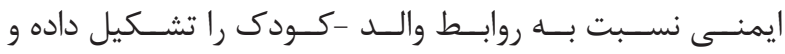

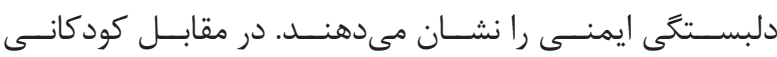

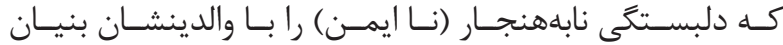

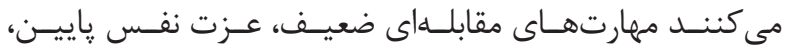

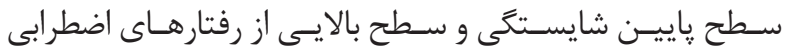

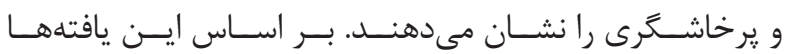

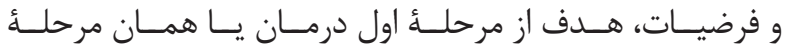

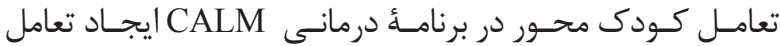

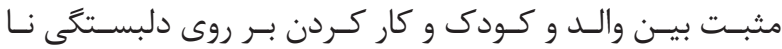

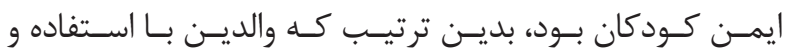

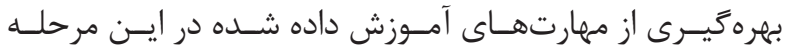

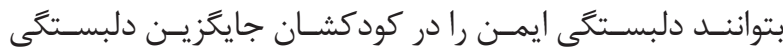

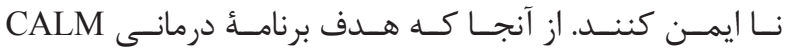

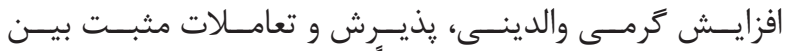

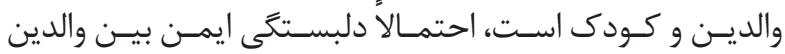

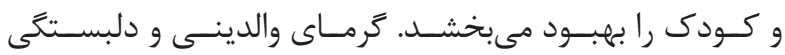

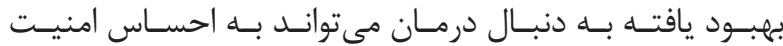

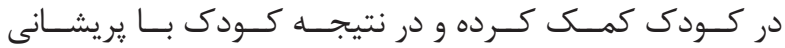

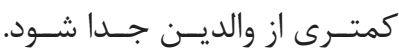

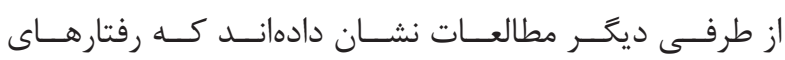

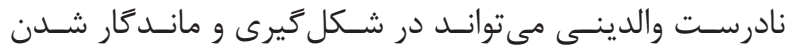

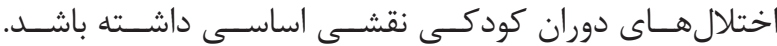

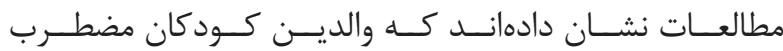

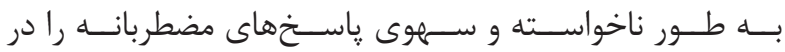

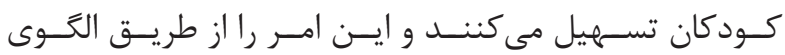

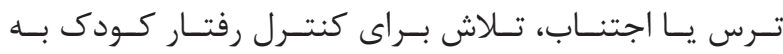

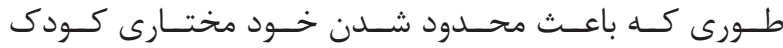

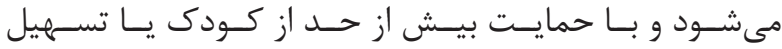

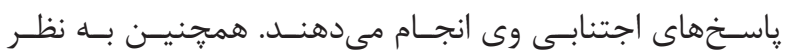

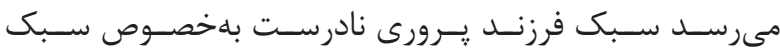

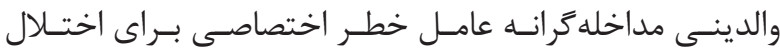

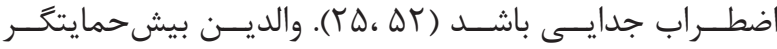

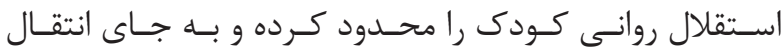




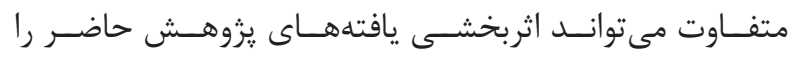

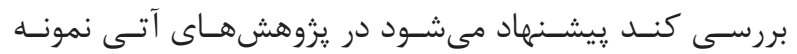

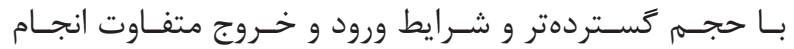

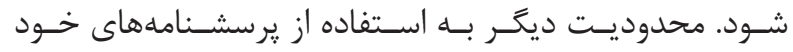

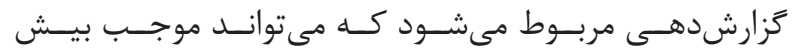

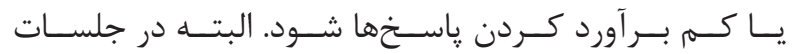

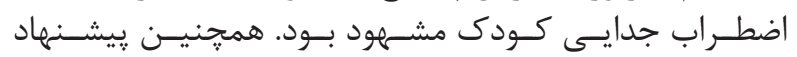

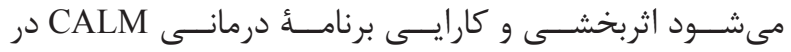

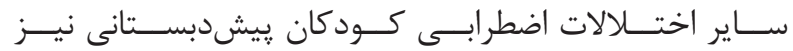

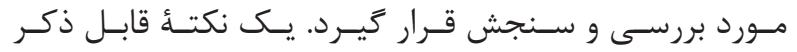

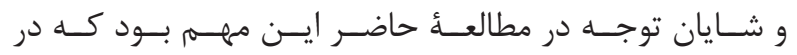

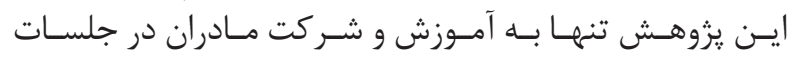

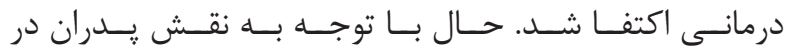

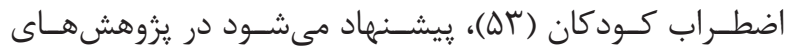

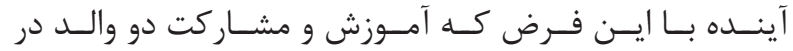

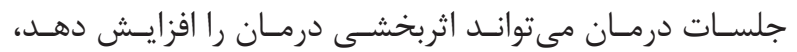

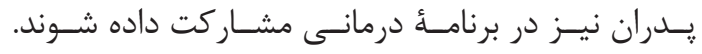

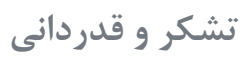

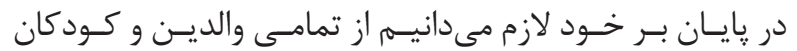

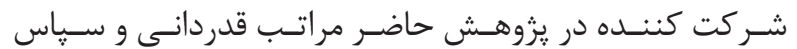

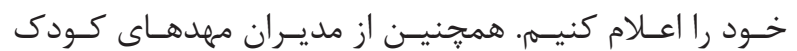

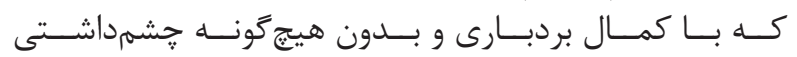

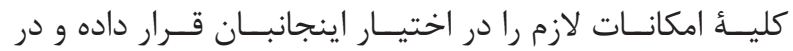

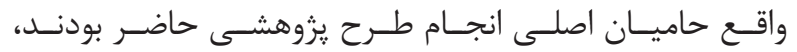

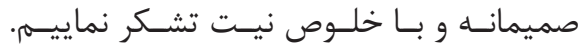

1. Cartwright-Hatton S, McNicol K, Doubleday E. Anxiety in a neglected population: prevalence of anxiety disorders in pre-adolescent children. Clin Psychol Rev. 2006; 26(7): 817-33.

2. Fisher JE, Donohue WT. Practitioner's Guide to Evidence-Based Psychotherapy. Dick-Niederhauser A, Silverman WK. Separation anxiety disorder. Springer US, 2006; p. 627-633.

3. Pincus DB, Eyberg SM, Choate ML. Adapting parent-child interaction therapy for young children with separation anxiety disorder. Educ Treat Children. 2005; 28(2):163-81.

4. Sadok Jims B. Synopsis of psychiatry: behavioral sciences and clinical psychiatry. Arjmand Publication. 2013.

5. Robert W. Child and adolescent psychopathology. Savalan Publication. 2009.

6. Pincus DB, Santucci LC, Ehrenreich JT, Eyberg SM. The implementation of modified parent-child interaction therapy for youth with separation anxiety disorder. Cogn

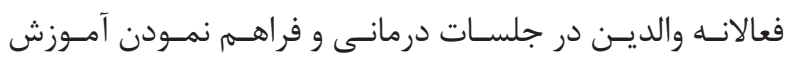

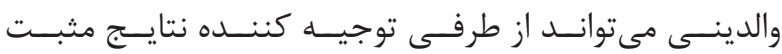

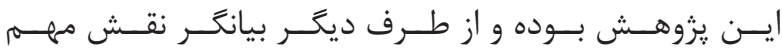

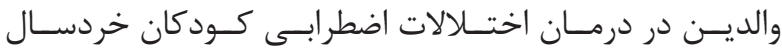

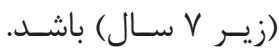

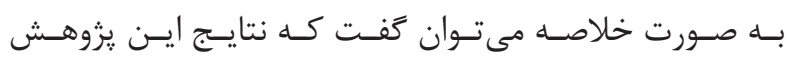

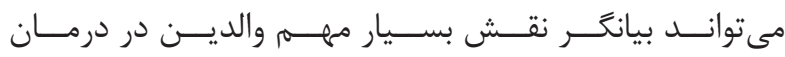

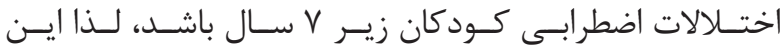

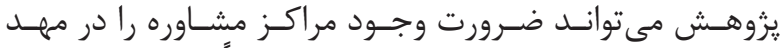

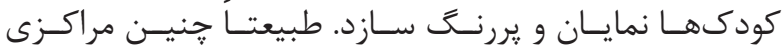

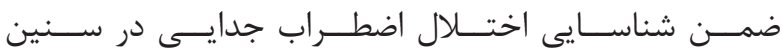

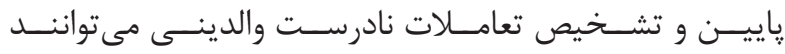

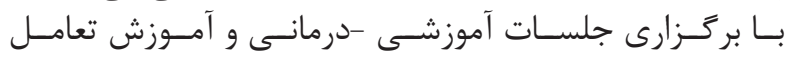

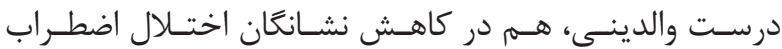

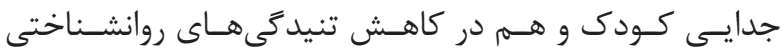

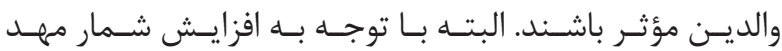

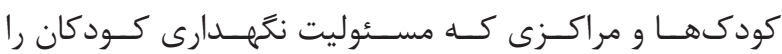

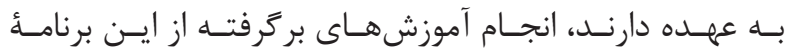

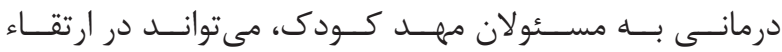

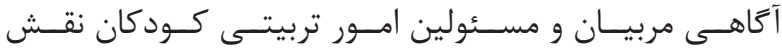

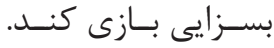

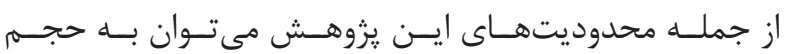

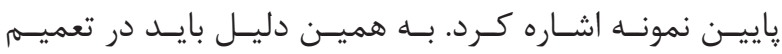

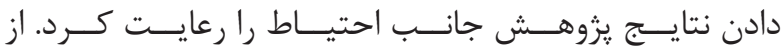

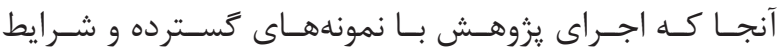

منابع

and Behav pract. 2008, (15): 118-25.

7. Dadsetan P. Morbid psychological transition from childhood to adulthood. Samt Publication. 2010.

8. Zolfaghari Motlagh M, Jazayeri AR, Khoshabi K, Mazahery MA, Karimloo M. Effectiveness of attachment based therapy on reduction of symptoms in separation anxiety disorder. IJPCP. 2009; 14(4): 380-88.

9. Eisen AR, Raleigh H, Neuhoff CC. The unique impact of parent training for separation anxiety disorder in children. Behav Ther. 2008; 39(2): 195-206.

10. Keller MB, Lavori PW, Wunder J, Beardslee WR, Schwartz CE, Roth J. Chronic course of anxiety disorders in children and adolescents. J Am Acad Child Adolesc Psychiatry. 1992; 31(4): 595-9.

11. Boden JM, Fergusson DM, Horwood LJ. Anxiety disorders and suicidal behaviours in adolescence and young adulthood: findings from a longitudinal study. Psycho Med. 2007; 37(3): 431-40.

12. Bittner A, Egger HL, Erkanli A, Costello JE, Foley 


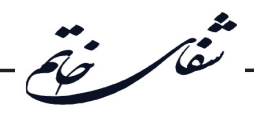

DL, Angold A. What do childhood anxiety disorders predict? J Child Psychol Psychiatry. 2007; 48(12): 1174-83.

13. Waters AM, Neumann DL, Henry J, Craske MG, Ornitz EM. Baseline and affective startle modulation by angry and neutral faces in 4-8-year-old anxious and non-anxious children. Biol. Psychol. 2008; 78(1): 10-9.

14. Costello EJ, Egger HL, Angold A. The developmental epidemiology of anxiety disorders: phenomenology, prevalence, and comorbidity. Child Adolesc Psychiatr Clin N Am. 2005; 14(4): 631-48.

15. Morris TL, March JS. Anxiety disorders in children and adolescents. $2^{\text {nd }}$. The Guilford Press. 2004.

16. Birrell L, Newton NC, Teesson M, Tonks Z, Slade T. Anxiety disorders and first alcohol use in the general population. Findings from a nationally representative sample. J Anxiety Disord. 2015; 31:108-13.

17. Teesson M, Hall W, Slade T, Mills K, Grove R, Mewton L, et al. Prevalence and correlates of DSM-IV alcohol abuse and dependence in Australia: findings of the 2007 national survey of mental health and wellbeing. Addiction. 2010; 105(12): 2085-94.

18. Kessler RC, Berglund $\mathrm{P}$, Demler O, Jin R, Merikangas KR, Walters EE. Lifetime prevalence and age-of-onset distributions of DSM-IV disorders in the national comorbidity survey replication. Arch gen psychiatry. 2005; 62(6): 593-602.

19. Doerfler LA, Toscano PF, Connor DF. Separation anxiety and panic disorder in clinically referred youth. J Anxiety Disord. 2008; 22(4): 602-11.

20. Nasirzadeh R, Roshan R. The effect of storytelling on aggression in six to eight-year old boys. Iranian Journal of Psychiatry and Clinical Psychology. 2010; 16(2):118-26.

21. Luby JL. Treatment of anxiety and depression in the preschool period. J Am Acad Child Adolesc Psychiatry. 2013; 52(4): 346-58.

22. Monga S, Rosenbloom BN, Tanha A, Owens M, Young A. Comparison of child-parent and parent-only cognitive-behavioral therapy programs for anxious children aged 5 to 7 years: short-and long-term outcomes. J Am Acad Child Adolesc Psychiatry. 2015; 54(2): 138-46.

23. Abdekhodaie MS, Sadeghi-Ordoubady A. Prevalence of separation anxiety in children and effect of cognitive behavioral play therapy on reduction it. Journal of Clinical Psycology. 2011; 4 (12): 51-8.
24. Bögels SM, Brechman-Toussaint ML. Family issues in child anxiety: attachment, family functioning, parental rearing and beliefs. Clin Psychol Rev. 2006; 26(7): 834-56.

25. Wood JJ. Parental intrusiveness and children's separation anxiety in a clinical sample. Child Psychiatry Hum Dev. 2006; 37(1): 73-87.

26. Muris P, Steerneman P, Merckelbach H, Meesters C. The role of parental fearfulness and modeling in children's fear. Behav Res Ther. 1996; 34(3): 265-8.

27. Hudson JL, Rapee RM. Parent-child interactions and anxiety disorders: An observational study. Behav Res Ther. 2001; 39(12):1411-427.

28. Affrunti NW, Ginsburg GS. Maternal overcontrol and child anxiety: The mediating role of perceived competence. Child Psychiatry Hum Dev. 2012; 43(1):102-12.

29. Fisak B Jr, Grills-Taquechel AE. Parental modeling, reinforcement, and information transfer: risk factors in the development of child anxiety? Clin Child and Famil Psychol Rev. 2007; 10(3): 213-31.

30. Hudson JL, Dodd HF, Bovopoulos N. Temperament, family environment and anxiety in preschool children. $\mathrm{J}$ Abnorm Child Psychol. 2011; 39(7): 939-51.

31. Bögels SM, Siqueland L. Family cognitive behavioral therapy for children and adolescents with clinical anxiety disorders. J Am Acad Child Adolesc Psychiatry. 2006; 45(2): 134-41.

32. Cartwright-Hatton S, McNally D, Field AP, Rust $\mathrm{S}$, Laskey B, Dixon CA, et al. A new parenting-based group intervention for young anxious children: results of a randomized controlled trial. J Am Acad Child Adolesc Psychiatry. 2011; 50(3): 242-51.

33. Silverman WK, Pina AA, Viswesvaran C. Evidencebased psychosocial treatments for phobic and anxiety disorders in children and adolescents. J Child Adolesc Psychol. 2008; 37(1): 105-30.

34. Chaffin M, Silovsky JF, Funderburk B, Valle LA, Brestan EV, Balachova T, et al. Parent-child interaction therapy with physically abusive parents: efficacy for reducing future abuse reports. J Consult Clin Psychol. 2004; 72(3): 500-10.

35. Chronis-Tuscano A, Lewis-Morrarty E, Woods KE, O'Brien KA, Mazursky-Horowitz H, Thomas SR. Parent-child interaction therapy with emotion coaching for preschoolers with attention-deficit/hyperactivity disorder. Cogn Behav Pract. 2014; 23(1): 62-78. 
36. Thomas R, Herschell AD. Parent-child interaction therapy: a manualized intervention for the therapeutic child welfare sector. Child Abuse Negl. 2013; 37(8): 578-84.

37. Lebowitz ER, Omer H, Hermes H, Scahill L. Parent training for childhood anxiety disorders: the SPACE program. Cogn Behav Pract. 2014; 21(4): 456-69.

38. McNeil C, Hembree-Kigin TL. Parent-child interaction therapy. Springer US. 2010.

39. Eyberg SM, Funderburk BW, Hembree-Kigin TL. McNeil CB, Querido JG, Hood KK. Parent-child interaction therapy with behavior problem children: One and two year maintenance of treatment effects in the family. Child Famil Behav Ther. 2001; 23(4):1-20.

40. Puliafico AC, Comer JS, Pincus DB. Adapting parent-child interaction therapy to treat anxiety disorders in young children. Child Adolesc Psychiatr Clin N Am. 2012; 21(3): 607-19.

41. Abbassi M, Neshat-Dust HT, Agha mohammadianshar-baaf HR. effectiveness of parent-child interaction therapy on reduction symptom's in separation anxiety disorder. Journal of Clinical Psychology. 2010; 2(2): 47-57.

42. Puliafico AC, Comer JS, Albano AM. Coaching approach behavior and leading by modeling: rationale, principles, and a session-by-session description of the CALM program for early childhood anxiety. Cogn Behav Pract. 2013; 20(4): 517-28.

43. Comer JS, Puliafico AC, Aschenbrand SG, McKnight $\mathrm{K}$, Robin JA, Goldfine ME, et al. A pilot feasibility evaluation of the CALM Program for anxiety disorders in early childhood. J Anx Disor. 2012; 26(1): 40-9.

44. Shamsaei F, Ozhan H, Jahangard L, Dehghani M, Soltanian AR. Attention deficit hyperactivity, oppositional defiant and conduct disorder in children of parents with bipolar disorder. Scientific Journal of Hamadan Nursing \& Midwifery Faculty. 2014; 22(3): 33-44.
45. Spence SH, Barrett PM, Turner CM. Psychometric properties of the Spence children's anxiety scale with young adolescents. J Anxiety Disord. 2003; 17(6):605-25.

46. Delavar A. Educational and psychological research. Tehran: Virayesh publication. 2014.

47. Makvand-Hosseyni SH, Kianersi F, Seyyedi-Andi SM. The effect of sandplay therapy on children's anxiety disorder. J New Thou Edu. 2012; 9(3):76-93

48. Fazeli Z, Shirazi E, Asgharnejad-Farid A, AfkhamEbrahimi A. Effectiveness of medication and combined medication and parent management training on visuocoonstructive, attentional, behavioral and emotional indicators of children with attention deficit/hyperactivity disorder. IJPCP. 2014; 19(4): 264-74.

49. Erermiş S, Bellibaş E, Ozbaran B, Büküşoğlu ND, Altintoprak E, Bildik T, et al. Temperamental characteristics of mothers of preschool children with separation anxiety disorder. Turk Psikiyatri Derg. 2009; 20(1): 14-21.

50. Tavakkolizadeh J, Bolhari J, Mehryar AH, Dezhkam M. Epidemiology of attention deficit and disruptive behavior disorder in elementary school children of Gonabad town. north east Iran (1996-1997). IJPCP. 1997; 3(2): 40-52.

51. Mohammadpour M. Effectiveness of CALM Program on reduction of Symptoms in Separation Anxiety disorder in preschool children. Msc thesis. Tabriz University. 2015.

52. Khanjani Z, Hashemi T, Peymannia B, Aghagolzadeh M. Relationship between the quality of mother-child interaction, separation anxiety and school phobia in children. Urmia Medical Journal. 2014; (25): 231-40.

53. Grossmann K, Grossmann KE, Fremmer-Bombik E, Kindler H, Scheurer-Englisch H, Zimmerman P. The uniqueness of the child- father attachment relationship: Fathers' sensitive and challenging play as a pivotal variable in a 16-year longitudinal study. Soc Dev. 2002; 11 (3): 301-37. 